\title{
Seguimento de Mulheres Laqueadas Arrependidas em Serviço Público de Esterilidade Conjugal
}

Follow-Up of Women who Sought Sterilization Reversal at a Public Infertility Clinic

Arlete Maria dos Santos Fernandes, Maurício De Souza Arruda, Marco Antônio Rocha Palhares Nei Danilo Benetti Junior, Cristiane Menabo Moreira

\begin{abstract}
RESUMO
Objetivo: analisar o seguimento de mulheres arrependidas que buscaram reversão da laqueadura tubárea em serviço público.

Métodos: foi realizado estudo retrospectivo das causas do arrependimento e dos resultados do tratamento em cima da população de mulheres com laqueadura tubárea atendida em Ambulatório de Esterilidade Conjugal, no periodo de junho de 1983 a junho de 1998. A análise dos dados foi descritiva.

Resultados: entre as 147 mulheres que procuraram o serviço, a maioria tinha idade entre 20 e 30 anos e 60\% delas havia sido submetida à laqueadura tubárea antes dos 25 anos de idade. Durante o acompanhamento, $54,4 \%$ das mulheres desistiram do tratamento, $15 \%$ foram desaconselhadas a seguir a investigação e somente 31 mulheres (21\%) foram submetidas à reanastomose tubárea. Quatorze mulheres engravidaram, e destas, nove $(6,1 \%)$ tiveram gestações a termo.

Conclusão: as mulheres que requerem laqueadura devem ser aconselhadas e esclarecidas sobre o caráter definitivo do método, tentando-se diminuir o arrependimento após o procedimento.
\end{abstract}

PALAVRAS-CHAVE: Infertilidade. Laqueadura tubárea. Reanastomose tubárea.

\section{Introdução}

A laqueadura tubárea é um método eficaz de anticoncepção. Devido a isso, muitos casais em todo o mundo o elegem para limitar o número de filhos. No Brasil, o número de mulheres que optam por este método é maior que na maioria dos países. Dados demográficos nacionais mostraram que entre mulheres entrevistadas nas faixas de idade de 25 a 29 anos e de 30 a 34 anos, a prevalência de laqueadura foi de $21,1 \%$ e $37,6 \%$, respectivamente. As taxas foram mais altas entre as mulheres em união estável, 26,9 e 42,7\%, para as mesmas faixas de idade ${ }^{1}$.

Entre as mulheres que optam pela laqueadura, muitas se arrependem, especialmente

Departamento de Tocoginecologia, Faculdade de Ciências Médicas, Pontificia Universidade Católica de Campinas Correspondência: Arlete Maria dos Santos Fernandes

Rua 14 de Dezembro, 421, ap. 21

13015-130 - Campinas - SP

e-mail: dramsf@acad.puccamp.br as mais jovens ${ }^{2-4}$. Em ambulatórios de esterilidade universitários, no período de 1980 a 1992, $23,5 \%$ das mulheres que haviam completado a investigação propedêutica tinham laqueadura tubárea ${ }^{5}$.

O sucesso da cirurgia de reversão é variável, segundo o parâmetro considerado, seja a permeabilidade tubárea restabelecida ou número de gestações entre o total de cirurgias realizadas. É claro que o número de gestações com nascidos vivos é o parâmetro verdadeiro de sucesso e essas taxas giram em torno de 30 a $48 \%$ das mulheres submetidas à reanastomose tubárea ${ }^{6,7}$. Um estudo de acompanhamento de 226 mulheres durante dois anos após cirurgia de reanastomose tubárea revelou $63 \%$ de gestações ${ }^{8}$. Entretanto, muitas mulheres que procuram os serviços de esterilidade desistem ou não conseguem realizar a cirurgia de reversão, e ainda, muitas das que são submetidas ao procedimento não conseguem uma gestação a termo.

Os objetivos deste trabalho foram avaliar as 
mulheres com laqueadura tubárea que procuraram o serviço por arrependimento e seu acompanhamento durante a propedêutica. Além disso, determinar quantas delas conceberam recémnascido a termo após o tratamento de reanastomose tubárea.

\section{Pacientes e Métodos}

O estudo foi de coorte retrospectivo. Foram revistos os prontuários das mulheres laqueadas que procuraram o Serviço de Esterilidade Conjugal no período de junho de 1983 a junho de 1998 . As variáveis estudadas foram a idade da mulher ao comparecer à primeira consulta e no momento da laqueadura, tempo decorrido entre a laqueadura e o arrependimento, persistência no seguimento do tratamento, reanastomose realizada ou não, permeabilidade pós-cirurgia, gestação pós-cirurgia e evolução da gestação.

As mulheres aceitas para a cirurgia de reversão, pelo protocolo do serviço, foram aquelas que não tinham sido laqueadas por indicação médica, apresentavam quadro clínico ou laboratorial compativel com o menacme, tinham idade de até 37 anos no momento do procedimento e haviam sido submetidas à laparoscopia prévia para verificação da possibilidade de reanastomose. O fator masculino foi afastado, antes da cirurgia, por meio de um espermograma normal.

Uma vez indicado o tratamento cirúrgico, a técnica realizada no serviço foi a de reanastomose tubárea uni ou bilateral segundo técnica microcirúrgica descrita inicialmente por Winston e Gomel e relatada por Inthraphuvasak, Pellicer e Bonilla-Musoles ${ }^{9}$. A verificação de permeabilidade pós-cirúrgica foi indicada nos casos de ausência de gravidez após no mínimo um ano da reanastomose e realizada mediante laparoscopia ou histerossalpingografia.

A análise dos dados foi descritiva. O estudo teve a aprovação do Comitê de Ética em Pesquisa da FCM-PUC-Campinas.

\section{Resultados}

Um total de 147 mulheres procurou o serviço para reversão da laqueadura no período estudado e a maioria delas $(73,4 \%)$ tinha entre 26 e 35 anos na época da primeira consulta. Cerca de $60 \%$ das mulheres tinham até 25 anos de idade no momento da laqueadura tubárea, um terço tinha entre 26 e 30 anos e somente uma mulher havia sido laqueada após os 35 anos de idade (Tabela 1).

Tabela 1 - Distribuição das faixas etárias das mulheres no momento da primeira consulta por esterilidade e quando da realização da laqueadura tubárea.

\begin{tabular}{crrrr}
\hline Faixas de idade & \multicolumn{3}{c}{ Primeira consulta } & \multicolumn{3}{r}{ Momento da laqueadura } \\
& $\mathbf{n}$ & $\%$ & $\mathbf{n}$ & $\%$ \\
\hline$<20$ & & - & 9 & 6,1 \\
$20-25$ & 11 & 7,5 & 79 & 53,8 \\
$26-30$ & 39 & 26,5 & 44 & 29,9 \\
$31-35$ & 69 & 46,9 & 14 & 9,5 \\
$>35$ & 28 & 19,1 & 1 & 0,7 \\
Total & 147 & 100,0 & 147 & 100,0 \\
\hline
\end{tabular}

Mais de metade das mulheres desistiu do tratamento após a primeira consulta ou com alguma propedêutica já realizada e 22 mulheres foram desaconselhadas a prosseguir na investigação por vários motivos (Tabela 2). Entre elas, 12 mulheres apresentavam idade maior de 37 anos, três tinham contra-indicação médica para nova gravidez, três tiveram colpocitologia alterada e foram encaminhadas à investigação, duas tinham parceiros oligospérmicos, uma mulher teve diagnóstico de falência ovariana prematura e uma estava sem parceiro no momento da marcação do procedimento.

Tabela 2 - Distribuição das mulheres segundo investigação de permeabilidade tubárea.

\begin{tabular}{lcc}
\hline Conduta de Investigação & $\mathbf{n}$ & $\mathbf{\%}$ \\
\hline Desistiram & 80 & 54,4 \\
Foram desaconselhadas & 22 & 15,0 \\
Foram investigadas & 45 & 30,6 \\
Total & 147 & 100,0 \\
\hline
\end{tabular}

Somente 45 mulheres foram submetidas à verificação de possibilidade cirúrgica, sendo que 13 delas não apresentavam condições para a recanalização. Entre estas, 10 haviam sido fimbrectomizadas e três apresentavam fator aderencial ou cotos insatisfatórios para a cirurgia de reversão. A reanastomose tubárea foi indicada para 32 mulheres. Foram submetidas à recanalização uni ou bilateral 31 mulheres e uma delas optou pelo tratamento em outro serviço.

Ocorreram 18 gravidezes em 14 mulheres $(9,5 \%)$. Apenas nove mulheres $(6,1 \%)$ conceberam 11 bebês a termo. Houve um óbito intra-uterino pré-termo e as quatro mulheres restantes tive- 
ram quatro gestações ectópicas e dois abortos (Tabela 3).

Treze mulheres não engravidaram durante o seguimento mínimo de um ano da reanastomose. Em 10 delas foi realizada pesquisa de permeabilidade, sendo que cinco apresentaram trompas pérvias após a cirurgia. Quatro mulheres perderam seguimento após a reanastomose e não temos informação se engravidaram ou não (Tabela 3).

Tabela 3 - Distribuição das mulheres segundo resultado da recanalização.

\begin{tabular}{|c|c|c|c|c|c|c|}
\hline \multicolumn{5}{|c|}{ Resultado } & \multicolumn{2}{|c|}{ Total } \\
\hline & A termo & Ectópica & Aborto & Óbito fetal & $\mathrm{n}$ & $\%$ \\
\hline \multirow[t]{2}{*}{ Engravidaram } & 9 & 3 & 1 & 1 & 14 & 45,2 \\
\hline & Bloqueio tubáreo & Trompa(s) permeável(is) & \multicolumn{2}{|c|}{ Permeabilidade não determinada } & & \\
\hline Não engravidaram & 5 & 5 & & & 13 & 41,9 \\
\hline \multicolumn{3}{|l|}{ Desconhecido } & & & 4 & 12,9 \\
\hline \multicolumn{3}{|c|}{ Total de mulheres submetidas à recanalização } & & & 31 & 100,0 \\
\hline
\end{tabular}

\section{Discussão}

A maioria das mulheres tinham idade entre 26 e 35 anos quando procuraram o serviço por esterilidade e $60 \%$ haviam sido submetidas à laqueadura até a idade de 25 anos. As mulheres deste estudo, em sua maior parte, haviam sido esterilizadas em idade muito jovem, no periodo de maior fertilidade da vida reprodutiva.

A escolha da laqueadura como método de planejamento familiar se deve a uma série de fatores, entre eles, o fato de as mulheres desconhecerem outros métodos reversiveis, a falta de oferecimento desses métodos como opção e o fato de muitas vezes os profissionais de saúde não esclarecerem que a laqueadura tubárea é um método definitivo de anticoncepção. Essa constatação fica clara em estudo caso-controle em nosso meio, onde as variáveis associadas ao arrependimento foram a idade inferior a 30 anos no momento da laqueadura, a falta de informação acerca da irreversibilidade do método e o menor conhecimento sobre os demais métodos contraceptivos antes da laqueadura ${ }^{10}$. A idade inferior a 30 anos no momento da laqueadura, como fator de risco para o arrependimento, também é citada em estudos internacionais ${ }^{11}$.

Apesar de se arrependerem do procedimento, metade das mulheres desistiu durante a propedêutica básica prévia à cirurgia e somente $21 \%$ foram submetidas à cirurgia de reversão. O fato de tantas mulheres desistirem do tratamento não quer dizer que tenham voltado a estar satisfeitas com o método que escolheram. Algum grau de arrependimento foi suficiente para um primeiro contato no serviço, entretanto, é possí- vel que existam dificuldades que as impeçam de persistirem no tratamento; uma delas pode ser a limitação pelo fator econômico. Mas esse abandono pode ser momentâneo e a mulher, no futuro em melhores condições financeiras ou resolvidos os fatores que a impeçam de prosseguir com o tratamento, pode retornar ao serviço.

Para a maioria das mulheres que realizaram a investigação, a possibilidade de reversão cirúrgica foi possivel. Com relação ao resultado da reversão, se fosse utilizado como parâmetro de sucesso cirúrgico a permeabilidade tubárea positiva após a cirurgia, de forma otimista, seria encontrado $61 \%$ de sucesso. Entretanto, deve-se ter em mente que nem sempre a permeabilidade tubárea conseguida na cirurgia é sinal de retorno da função tubárea, sendo que muitas mulheres não engravidam mesmo tendo recuperado a permeabilidade das trompas. Quando as trompas reconstituídas não recuperam sua função, a alternativa de tratamento seria a reprodução assistida por meio da técnica de fertilização in vitro e transferência de embriões (FIV-TE). Entretanto, o alto custo desse procedimento torna inviável e limitante esse tratamento para a maioria das mulheres em nosso meio.

Além disso, mesmo em caso de gravidez após a reanastomose, existe maior chance de gestação ectópica ou, algumas vezes, a gestação terminará em aborto. Pelo resultado apresentado, cinco das 14 mulheres que engravidaram tiveram resultado de insucesso.

A taxa de sucesso do tratamento, se considerarmos o número de gestações em relação ao total de cirurgias realizadas, foi de $45 \%$ e diminui para $29 \%$ se considerarmos somente as mulheres que tiveram um bebê saudável ao final da ges- 
tação. Nossas taxas encontradas são compativeis com a literatura. Entretanto, queremos ressaltar que, quando tomamos como parâmetro o número de gestações a termo em relação ao total de mulheres laqueadas que procuraram tratamento $(9 /$ 147), encontramos uma taxa de sucesso de $6 \%$. Este resultado está em acordo com outro estudo, realizado com 394 mulheres laqueadas que procuraram tratamento em um serviço público em nosso meio, no qual são relatadas 13 gestações, o que correspondeu a 3,3\% do total de mulheres atendidas $^{12}$. Essas são as taxas de sucesso que podemos oferecer, quando trabalhamos no setor público, às mulheres laqueadas arrependidas que chegam procurando tratamento.

No Brasil, a laqueadura tubárea é tema complexo. Sua prevalência está associada a variáveis sociodemográficas e correlaciona-se intimamente com a prevalência de parto cesáreo ${ }^{13}$. Em vista desse contexto, numa tentativa de normatizar a indicacão de métodos contraceptivos no país, foi promulgada a lei 9.263 de 12 de janeiro de 1996 que regulamenta o Parágrafo $7^{\circ}$ do artigo 226 da Constituição Federal que trata do planejamento familiar e estabelece penalidades e dá outras providências ${ }^{14}$.

O fato de existir uma lei específica para o planejamento familiar é, sem dúvida, positivo. Entretanto, chama a atenção o artigo $10^{\circ}$ que determina que somente é permitida a esterilização voluntária nas seguintes situações: "em homens $e$ mulheres com capacidade civil plena e maiores de vinte e cinco anos de idade ou, pelo menos, com dois filhos vivos...". Isso quer dizer que homens e mulheres com mais de 25 anos sem filhos podem ser definitivamente esterilizados. Da mesma forma, se tiverem pelo menos dois filhos, podem ser esterilizados a partir dos 21 anos. Essa questão faz refletir e supor que, com o passar dos anos, haverá um aumento do número de mulheres laqueadas arrependidas. Se por um lado existem situações nas quais o método definitivo pode ser indicado para pessoas jovens, por outro é necessária uma avaliação médica criteriosa, consciente e particularizada de cada caso, mesmo diante da permissividade da lei.

A lei também proíbe a realização da laqueadura durante o periodo de parto e puerpério. $\mathrm{E}$ determina, inclusive, que a partir do momento da opção pelo método, o procedimento somente possa ser realizado após decorridos sessenta dias, dando-se ampla assistência ao casal, durante esse período, com relação aos métodos reversiveis. A laqueadura passa a ser uma questão médica e social controversa na medida em que o procedimento requer uma internação fora do periodo do parto e puerpério para que seja realizado, o que gera um ônus ao serviço público. E quando optamos pela esterilização de mulheres jovens, geramos um maior gasto futuro, no momento do arrependimento.

Somados todos esses fatores, concluímos que devemos considerar a laqueadura tubárea como método de anticoncepção definitivo para a população. Atentos à situação, os médicos devem alertar as mulheres que desejarem a laqueadura como método de anticoncepção, quanto ao caráter irreversivel do procedimento. Além dos médicos, os profissionais de saúde que acompanham mulheres em idade reprodutiva poderão estimular a adoção de métodos anticoncepcionais reversiveis e eficazes, especialmente para as mais jovens, o que certamente possibilitará diminuição do número de mulheres arrependidas nos ambulatórios de esterilidade.

\section{SUMMARY}

Purpose: to analyze the follow-up of regretful sterilized women who sought sterilization reversal at a public service. Method: a retrospective study was carried out with a population of sterilized women who attended the infertility clinic during the period from June 1983 to July 1998. The analysis of the data was descriptive.

Results: among the 147 women who sought the infertility clinic, most were 20 to 30 years old and $60 \%$ were sterilized at the age of 25 years. During follow-up, $54.4 \%$ of the initial patients gave up treatment, $15 \%$ were discouraged to continue the investigation and only 31 (21\%) underwent tubal anastomosis. Fourteen women became pregnant and nine $(6.1 \%)$ of them had term gestation.

Conclusion: women who request sterilization should be counseled and instructed about the definitive feature of this method in order to reduce regret after sterilization.

KEY WORDS: Infertility. Sterilization, tubal. Anastomosis, tubal.

\section{Referências}

1. PNDS. Pesquisa Nacional de Demografia e Saúde. Rio de Janeiro: BEMFAN; 1996. p.52-61.

2. Leader A, Galan N, George R, Taylor PJ. A comparison of definable traits in women requesting reversal of sterilization and women satisfied with sterilization. Am J Obstet Gynecol 1983; 145:198-202. 
3. Boring CC, Rochat RW, Becerra J. Sterilization regret among Puerto Rican women. Fertil Steril 1988; 49:973-81.

4. Bahamondes L, Petta CA, Faúndes A, Bedone A. Significado do recente aumento do número de solicitantes de reversão de laqueadura em um serviço de esterilidade. Femina 1992; 20:360-2.

5. Fernandes AMS, Bahamondes L. Incidência dos fatores etiológicos da esterilidade conjugal nos hospitais universitários de Campinas. Rev Bras Ginecol Obstet 1996; 18:29-36.

6. Passos EP, Freitas F, Cunha Filho JS, et al. Cirurgia tubária para infertilidade. Reprod Clim 1996; 11:25-7.

7. Gillett WR, Clarke RH, Herbison GP. First and subsequent pregnancies after tubal microsurgery: evaluation of the fertility index. Fertil Steril 1997; 68:1033-42.

8. Dubuisson JB, Chapron C, Nos C, Morice P, Aubriot FX, Garnier P. Sterilization reversal: fertility results. Hum Reprod 1995; 10:1145-51.

9. Inthraphuvasak J, Pellicer A, Bonilla-Musoles F. Microcirugía en la porción proximal de la trompa, anastomosis del segmento medio y reversibilidad de la esterilización. In: Inthraphuvasak $\mathrm{J}$, Pellicer A, Friedberg V, Bonilla-Musoles F, editores. Microcirugía Tubárica. $1^{\text {a }}$ ed. Barcelona: Ed. Jims; 1984. p.192-6.

10. Hardy E, Bahamondes L, Osis MJ, Costa RG, Faúndes A. Risk factors for tubal sterilization regret, detectable before surgery. Contraception 1996; 54:159-62.

11.Grubb GS, Peterson HB, Layde PM, Rubin GL. Regret after decision to have a tubal sterilization. Fertil Steril 1985; 44:248-53.

12.Petta CA, Bahamondes L, Hidalgo M, Faúndes A, Bedone AJ, Faúndes D. Follow-up of women seeking sterilization reversal: a Brazilian experience. Adv Contracept 1995; 11:157-63.

13. Faúndes A, Costa RG, Pádua KS, Perdigão AM. Associação entre prevalência de laqueadura tubária e características sócio-demográficas de mulheres e seus companheiros no Estado de São Paulo, Brasil. Cad Saúde Pública 1998; 14 Supl 1:49-57.

14.Ferriani RA. A lei da esterilização cirúrgica. Reprod Clim 1997; 12:161-2.

\section{Inscrições:16 de Abril a 31 de Maio de 2001}

\section{Informações na Federada do seu Estado}

\section{Prova: \\ 26 de Agosto de 2001}

